\title{
Nutraceuticals as a New Approach in Management of Diabetic Nephropathy
}

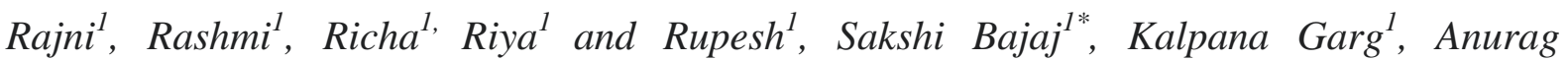 \\ Bhargaval $^{l}$ \\ ${ }^{1}$ CH. Devi Lal College of Pharmacy, Jagadhari-135003, Haryana
}

\section{Email: sakshibajaj2012@gmail.com}

\begin{abstract}
Diabetic nephropathy (DN), a fatal diabetic complication, is a prime reason of end-stage renal disease (ESRD), which is pathologically identified by thickened tubular basal and glomerular membranes, amassed extracellular matrix (ECM), and developing meningeal hypertrophy. Various deformities in the signaling pathway can interact to give rise to these pathologic activities in DN. In spite of the accessibility of numerous approaches to prevent these metabolic changes, which include proper diet, exercising regularly, control of weight and control of drugs, epidemiological data are observing the growing tendency of the complication, indicating both the multifactorial nature of these disorders as well as the scarce conformance of patients to begin strategies. A number of Nutraceuticals utilized in clinical practice were shown to aim the pathophysiology of diabetes mellitus, metabolic disorder and their complications and to favorably modify numerous biochemical and clinical endpoints. These compounds comprise of antioxidants, vitamins like vitamin $C, E, D$; Omega 3 fatty acids, alpha lipoic acid (ALA), dietary fibers, flavonoids, phytoestrogens and minerals like chromium, magnesium. Various areas of concern prevail with regard of the use of nutraceuticals and dietary supplements in this setting, inclusive of standardization of products, potential side effects, definition of dosing regimen, interaction of drugs and need for evidence based interactions.
\end{abstract}

Keywords: Diabetic Nephropathy, Nutraceuticals, Pathogenesis, Complications, omega-3fatty acid, vitamin D, dietary fibers, curcumin, resveratrol, quercetin, berberine, vitamin C.

\section{Introduction}

Diabetes is a non-communicable metabolic disorder that arises due to hormonal imbalance such as insulin, glycogen. Two types of diabetes Type 1 diabetes alias insulin dependent diabetes mellitus (IDDM), is caused by lack of insulin secretion by beta cells of the pancreas and Type 2 diabetes alias non-insulin dependent diabetes mellitus (NIDDM), is caused by decreased sensitivity of target tissues to insulin [1]. Diabetes is widely recognized as an emerging epidemic that has a cumulative impact on almost every country, age group, and economy across the world. According to the International Diabetes Federation, it was found that in 2015, approximately 415 million people were suffering from diabetes worldwide, and this number is expected to exceed 640 million by the year 2040. In 2020, according to the International Diabetes Federation (IDF), 463 million people have diabetes in the world and 88 million people in the Southeast Asia region. Of this 88 million people, 77 million belong to India. According to the 2019 National Diabetes and Diabetic Retinopathy Survey report released by the Ministry of Health and Family Welfare, the prevalence was found to be $11.8 \%$ in people over the 
age of 50. The prevalence of diabetes is $6.5 \%$ and prediabetes $5.7 \%$ among the adults below the age of 50 years, according to the DHS survey [2]. There are various complications that are associated with long-term damage and failure of various organ systems. Diabetes complications are common in patients with type 2 diabetes. The chronic complications of diabetes are broadly categorized into microvascular and macrovascular, with the former having much higher ascendency than the latter. Microvascular complications include neuropathy, nephropathy, and retinopathy, while macrovascular complications consist of cardiovascular disease, stroke, and peripheral artery disease (PAD) [3] Diabetic Nephropathy (DN) alias Diabetic Kidney Disease (DKD) is a dreadful impediment of type 1 and type 2 diabetes mellitus (DM) associated with the kidneys [4]. DN is a medical condition depicted by glomerular hypertrophy, protein in urea, diminished glomerular filtration and kidney fibrosis which further bring about deprivation of kidney function [5]. DN is proclaimed to eventuate in 20\%$50 \%$ of individuals living with DM and is the prevailing root of End Stage Kidney Disorder (ESKD) [6]. Indications of general DN is perpetual albumin in urea with retinopathy and no authentication of alternative kidney disorder [7]. The possibility of evolving DN differs between individuals and it relies not only on span of DM but is influenced by numerous other agents. Type 2 DM reports about $90 \%$ of diabetes throughout the world, so the large number of individuals who suffered from DN is because of type 2 DM [8]. Diabetic nephropathy can be rectified by correcting these parameters like Cardiovascular risk alleviation, maintaining glycaemic level, maintaining blood pressure, Blocking of Rennin Angiotensin Aldosterone System (RAAS), Changing the way of life [9]. Some of the allopathic drugs used in the treatment of diabetic nephropathy are Keresan, Benzoctamine, Robotisation, Telmisartan, Rapamycin, Fenofibrate, Liseran, and Barnidipine [5]. Allopathic drugs may cause long term side effects but still used widely. Nowadays, nutraceuticals have received high advantage due to potential nutritional, safety, and therapeutic effects. The nutraceuticals are used widely, also may be due to the fact that many of them are easily available, inexpensive and available in small doses. They usually do not cause repulsive side effects [10]. The purpose of this review is to summarize nutraceuticals as a current approach in the treatment of Diabetic Nephropathy. According to our hypothesis if the combination of two or more nutraceuticals can be more effective as compared to single nutraceutical. Further clinical studies must be done to clarify the effect of combination of nutraceuticals.

\section{Diabetic Nephropathy Pathogenesis}

There are several pathways that may cause Diabetic nephropathy (DN), such as hyperglycemiainduced renal hyper filtration and renal injury, AGEs-induced increased oxidative stress, activated PKC-induced increased production of cytokines, chemokine's, and different inflammatory and apoptotic signals. Among all, oxidative stress has been suggested to play a major role in the propagation of DN. It stimulates several signaling pathways involved in DN, like AGEs, PKC cascade, JAK/STAT signaling, MAPK, m-TOR, and SMAD [41].

Polyol Pathway is a process that increases the ratio of NADH/NAD and may result in both oxidative stress and activation of protein kinase C. The Polyol pathway involves two enzymes. (I) Aldose Reductase (AR), reduces glucose to sorbitol by using NADPH as a co-factor, and (ii) sorbitol dehydrogenase (SDH) that converts sorbitol to fructose with the aid of its cofactor NAD+. Then, the excess sorbitol is oxidized to fructose [42]. This flux of glucose through the Polyol pathway would increase Advance Glycation End Products (AGE) formation that may result in oxidative stress [43].

AGEs pathways (Advance Glycation End Products): - AGEs accumulate at site of micro vascular injury in diabetes, including the kidney [44], the retina and within the vasculature [45]. AGE stimulate 
activation and expression of IL-6 and TGF $\beta 1$ via NF-kB dependent pathways [45]. The main site for reabsorption of filtered AGEs is proximal tubule [46]. TGF- $\beta 1$ expression is significantly linked to accumulation of AGEs in the kidney [47]. AGEs lead to the transcriptional up-regulation of TGF- $\beta 1$, possibly via PKC or oxidative stress. In experimental diabetes, oxidative stress is increased in proportion to the accumulation of AGEs [48]. This pathway can also lead to enhanced formation of free radicals both directly through catalytic sites in their molecular structure [49]. The generation of superoxide may be enhanced due to mitochondrial dysfunction induced by AGEs and carbonyl intermediates [50]. AGE also contribute to the release of pro-inflammatory cytokine and growth factor expression and adhesion molecule such as VEGF and CTGF, TGF- $\beta 1$, IGF-1, PDGF, TNF- $\alpha$, IL-1 $\beta$, and IL-6 [44,50].

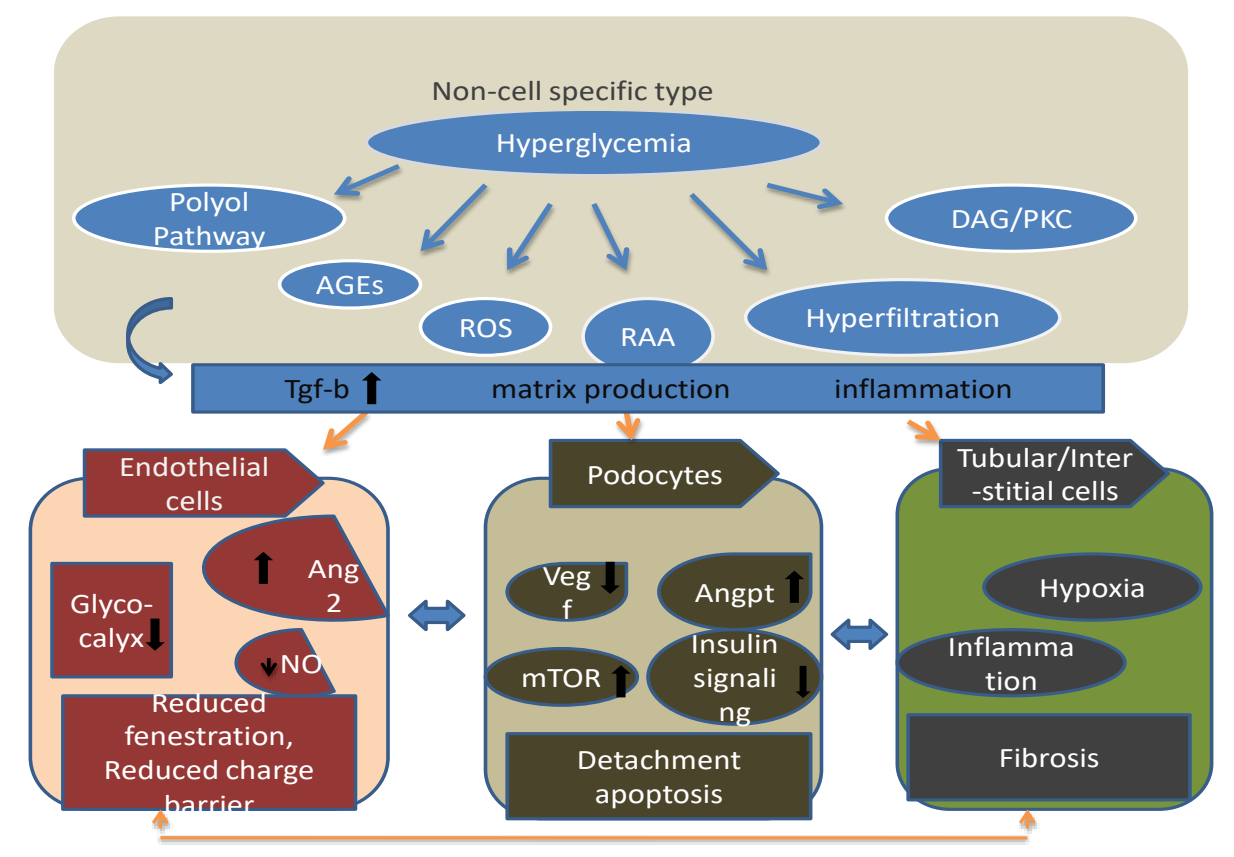

Figure1. Schema outlining various factors in the Pathogenesis of Diabetic Nephropathy

AGEs $=$ advanced glycation end products; $\mathrm{ROS}=$ reactive oxygen species; RAA $=$ Renin angiotensin aldosterone system; $\mathrm{DAG}=$ diacylglycerol; $\mathrm{PKC}=$ protein kinase $\mathrm{C} ; \mathrm{NO}=$ nitrogen oxide; $\mathrm{mTOR}$ =mammalian target of rapamycin; VEGF= vascular endothelial growth factor;

Protein kinase C pathway: - Out of eleven isoforms of PKC, nine PKCs are activated by DAG, which is formed from excess glyceraldehydes-3-phosphate. Increased glucose concentration leads to increase the amount of DAG, which activates PKC. PKC activation further leads to changes in renal blood flow [51], by decreasing production of NO [52], meningeal expansion, albuminuria and increases GFR, increases pro-inflammatory gene expression and vascular permeability in many models of experimental diabetes [53]. Activation of PKC may be responsible for the increased expression of ECM molecules both directly and through TGF- $\beta 1$ over expression.

Hexamine Pathway: - The Hexokinase is an essential enzyme for conversion of fructose-6-phosphate into glucosamine-6-phosphate. The Glutamine: Fructose-6-Phosphateamidotransferase (GFAT) is the rate-limiting enzyme of this pathway. Both high glucose and Angiotensin II activates the GFAT promoter in meningeal cells (MC) [54] and further that may enhance flux through the Hexokinase. Over activation of GFAT in MC leads to increase both TGF- $\beta$ and fibronectin expression [55]. 


\section{Involvement of pro-inflammatory cytokines in diabetic nephropathy: -}

TNF-a: - Renal hyper-filtration and hypertrophy are the some of the early changes that occur in the kidney, which seems to be due to inflammation particularly by TNF-a. For TNF-a to exert its actions, two mediators responsible i.e., receptors [56] TNFR1 and TNFR2. As TNF-a binds to these mediators and results in the formation of the TNFR associated death domain (TRADD) [57].IL-6 act by binding to the membrane-bound IL6R and gp130 signal-transducing chain. This result in the auto phosphorylation as a result of which both JAKs closer to each other to phosphorylate one another inducing an intracellular signal and furthers and activates transcription factors, signal transducer and activator of transcription (STAT)-3. This activation leads to the growth and proliferation of meningeal cells [58].

Once released, IL-6 binds to the membrane-bound IL6R and gp130 signal-transducing chain. This result in the auto phosphorylation and change in the conformational structure of Janus kinase (JAK) which brings both JAKs closer to each other to phosphorylate one another inducing an intracellular signal. This further phosphorylate and activates transcription factors, signal transducer and activator of transcription (STAT)-3. Consequently, this leads to the growth and proliferation of meningeal cells and subsequently a similar outcome as IL-1 as described previously [59].

Role of Oxidative Stress in Diabetic Nephropathy Hyperglycemia-induced oxidative stress has been suggested as the common mechanism causing the cell damage seen in diabetic complications [60]. Oxidative stress plays a major role in pathological changes of the kidney [61]. An imbalance between Reactive Oxygen Species (ROS) and intracellular antioxidants leads to Oxidative stress [62]. In Hyperglycemia overproduction of superoxide induced by mitochondrial electron transfer chain is the major molecular mechanisms for diabetes. Furthermore, increased NADPH oxidase activity leads to production of ROS in diabetic nephropathy [63]. Moreover, activation of PKC pathway leads to the production of ROS in diabetes which is attenuated by PKC inhibitors.

\section{Genetic mechanisms leading to diabetic nephropathy}

Angiotensin-converting enzyme (ACE): - The dysfunctional ACE gene can cause toxicity and fibrosis of blood vessels by the increasing aldosterone level [65]. It was found that by the activation of the Smad2-dependent TGFb1 pathway the aldosterone aids in the production of the extracellular matrix protein, fibronectin by glomerular meningeal [66].

FRMD3: - Another gene associated with DN is the FRMD3. An SNP, rs1888747 located on chromosome 9 in the promoter region of FRMD3 is strongly associated with DN [67] Martini and colleagues (2013) suggested that FRMD3 operates in conjunction with bone morphogenetic protein (BMP) signaling pathway [68]. BMP is part of the TGFb1 super-family plays a part in kidney development, chemo taxis, and cell differentiation and regulates apoptosis of various adult cell types such as hematopoietic, epithelial, neuronal and mesenchymal cells [69]. The rs1888747 SNP in the promoter region of FRMD3 is considered to suppress the activity of FRMD3, which prevents the activation of BMP and the depletion of BMP-mediated renal protection in patients with diabetes [70]. FRMD3/BMP inhibition can result in albuminuria and increase in fractional meningeal area. For example, BMP7 agonists and antagonists like kielin/chordin-like protein and gremlin respectively have been reported in patients with DN and suppressed the expression of BMP7 and its agonists have shown to increase profibrotic activity in DN [41, 42]. 


\section{Nutritional Interventions Targeting the Pathogenesis of Diabetes Mellitus: -}

\section{Omega-3 fatty acids:}

Omega-3 polyunsaturated fatty acids (n-3 PUFAs), including the essential fatty acids $\alpha$-linoleic acid (ALA) and the long chain fatty acids eicosapentaenoic acid (EPA) and docosahexaenoic acid. It is an (DHA), obtained from plants and marine life. It gains great interest in the health benefits among the many chronic diseases in which n-3 PUFA intake can be improved, there is a large literature that discusses the therapeutic potential associated with type 2 diabetes [73]. The mechanism by which omega- 3 fatty acids reduce proteinuria is not yet clear. There is evidence that omega- 3 fatty acids may affect renal hemodynamic [74]. The effect of omega-3 fatty acids on increasing proteinuria may exceed hemodynamic parameters. One hypothesis is that omega-3 fatty acids may reduce urinary protein excretion via anti-inflammatory effects and oxidative stress [75]. O3FA also may reduce proteinuria in patients with chronic glomerular disease and delayed immunoglobulin A (IgA) nephropathy [76]. Because of its anti-inflammatory action, O3FA is thought to prevent kidney damage. An anonymous model study has shown that O3FA formulations reduce renal inflammation and fibrosis [77]. Experimental Diabetic Nephropathy Garman et al. studied the effect of omega-3 polyunsaturated fatty acids administered to male streptozotocin-induced diabetic Sprague-Dawley rats for 30 weeks. Mice that consumed polyunsaturated fatty acids high in nonomega-3 had significantly less albuminuria, high blood pressure, glomerulosclerosis, and interstitial tubular fibrosis. In addition, the increase in type IV and this collagen, IL-6, MCP-1, TGF and the macrophage marker CD68 was reduced. The researchers concluded that omega-3 polyunsaturated fatty acids significantly reduce kidney damage caused by diabetes [78].

\section{Berberine: -}

Berberine (BBR; [C20H18NO4] +).an isoquinoline alkaloid isolated from the rhizome of Coptidis, cortex philodendra and Berberis vulgaris, has long been used widely as an Eastern medicine for the treatment of gastroenteritis and secretarydiarrhea [79-82]. Recent studies have illustrate that berberine has various pharmacological activities, including lowering blood glucose, regulating blood lipids and reducing inflammation in addition to its antioxidant activity. These findings suggest that berberine has applications as a therapeutic drug for diabetic nephropathy, and has significant research value [83]. In addition, BBR is well characterized as having a Reno protective effect on the development of DN [84, 85]. However, the possible mechanisms have not been fully established. The purpose of this article is to investigate the Reno protective mechanisms of berberine in diabetic nephropathy and highlight the importance of berberine as a potential therapeutic reagent for diabetic nephropathy treatment [83]. Inflammation, fibrosis, and lipid abnormalities are the main causes of the pathogenesis of diabetic kidney damage in type 2 diabetes.[86] Berberine (BBR) has been reported to have beneficial effects in diabetic nephropathy, but its mechanism of action is unclear. This study aims to determine the therapeutic mechanism of LBW in a rat model of type 2 diabetic nephropathy induced by a high-fat streptozotocin regimen and low-dose injection. Diabetic mice were given tubes with or without BBR for 20 weeks and examined by serological, 24-hour proteinuria, histology, immunohistochemistry, and molecular tests [86]. The results showed that LBW treatment significantly reduced serum sugar and lipids, inhibited urinary tract albumin excretion, and attenuated histological kidney damage in diabetic rats. Berberine treatment also suppresses renal inflammation associated with inactivation of a key factor that increases kappa through an activated B-cell signaling light chain. As a result, the up regulation of pro-inflammatory cytokines (interleukin- $1 \beta$, tumor necrosis factor- $\alpha$ ) and chemokine's (protein of chemotactic monocytes-1) is blocked. In addition, BBR treatment inactivates growth 
factor- $\beta$ / Smad3 transforming signal modification and suppresses renal fibrosis, including the expression of fibronectin, collagen I, and collagen IV. This study shows that BBR is a diabetes remedy. Relief of type 2 nephropathy consists in suppressing the kappa-nuclear potentiator of the activated inflammatory light chain of B cells and altering the signaling pathway of growth factor- $\beta$ / Smad3 [85]. 
Table no. 1 List of Nutraceuticals used in Diabetic Nephropathy

\begin{tabular}{|c|c|c|c|c|}
\hline Nutraceuticals & Source & $\begin{array}{l}\text { Mechanism of action } \\
\text { in DN }\end{array}$ & Other activities & References \\
\hline $\begin{array}{l}\text { Omega-3 fatty } \\
\text { acids }\end{array}$ & $\begin{array}{l}\text { obtained from } \\
\text { plants and marine } \\
\text { life }\end{array}$ & $\begin{array}{l}\text { may reduce urinary } \\
\text { protein excretion } \\
\text { through anti- } \\
\text { inflammatory effects } \\
\text { and oxidative stress }\end{array}$ & $\begin{array}{l}\text { Anti-inflammatory, anti- } \\
\text { thrombotic, decrease TGs, } \\
\text { reduce blood clotting time }\end{array}$ & Clark WF et al \\
\hline Berberine & $\begin{array}{l}\text { isoquinoline } \\
\text { alkaloid isolated } \\
\text { from the rhizome } \\
\text { of Berberis } \\
\text { vulgaris }\end{array}$ & $\begin{array}{l}\text { Reno protective by } \\
\text { reducing fibrosis, } \\
\text { Inflammation, , and } \\
\text { lipid abnormalities }\end{array}$ & $\begin{array}{l}\text { lowering blood glucose, } \\
\text { regulating blood lipids and } \\
\text { reducing inflammation }\end{array}$ & Han $\mathbf{J}$ et al \\
\hline Dietary fibers & $\begin{array}{l}\text { obtained from } \\
\text { plant products of } \\
\text { polysaccharides, } \\
\text { oligosaccharides, } \\
\text { lignin }\end{array}$ & $\begin{array}{l}\text { Modulation of gut } \\
\text { micro biota, } \\
\text { enrichment of SCFA- } \\
\text { producing bacteria }\end{array}$ & $\begin{array}{l}\text { increasing body weight, } \\
\text { lowering blood cholesterol } \\
\text { levels and, coronary artery } \\
\text { disease, dyslipidemia and } \\
\text { hypertension }\end{array}$ & JULIE M J et al \\
\hline Quercetin & $\begin{array}{l}\text { Cabbage, onions, } \\
\text { fruits, apple, red } \\
\text { wine, broccoli, } \\
\text { cherries, tea and } \\
\text { red wine etc. }\end{array}$ & $\begin{array}{l}\text { powerful antioxidant } \\
\text { properties potent ROS } \\
\text { scavenger and may } \\
\text { reduce the risk of } \\
\text { cardiovascular disease }\end{array}$ & $\begin{array}{l}\text { prevents various diseases } \\
\text { such as osteoporosis, some } \\
\text { forms of cancer, tumors, } \\
\text { lungs, }\end{array}$ & Chen T.C. et al \\
\hline $\begin{array}{l}\text { Alpha lipoic } \\
\text { acid [ALA] }\end{array}$ & $\begin{array}{l}\text { spinach, then } \\
\text { broccoli, } \\
\text { tomatoes, garden } \\
\text { peas and rice } \\
\text { bran }\end{array}$ & $\begin{array}{l}\text { Powerful ROS } \\
\text { scavenger, renew } \\
\text { endogenous } \\
\text { antioxidants }\end{array}$ & $\begin{array}{l}\text { the treatment of } \\
\text { Alzheimer's disease, } \\
\text { cancer, diabetic } \\
\text { complications, multiple } \\
\text { sclerosis, diabetes, obesity }\end{array}$ & Oza M.J. et al \\
\hline Vitamin D & $\begin{array}{l}\text { flesh of fatty fish } \\
\text { and fish liver oils } \\
\text { egg yolks, } \\
\text { cheese, and beef } \\
\text { liver }\end{array}$ & $\begin{array}{l}\text { encouraging insulin } \\
\text { production, restrict } \\
\text { renin production, and } \\
\text { stimulating macro - } \\
\text { phage cathelicidin } \\
\text { development }\end{array}$ & $\begin{array}{l}\text { Renal protective by } \\
\text { proteinuria, antifibrosis, } \\
\text { anti-inflammatory, and } \\
\text { Inhibiting podocyte } \\
\text { devastation }\end{array}$ & $\begin{array}{l}\text { Holick, M. F. et } \\
\text { al }\end{array}$ \\
\hline Flavonoids & $\begin{array}{l}\text { fruits, vegetables, } \\
\text { tea, cocoa and } \\
\text { wine, onion, tea }\end{array}$ & Anti- oxidant & $\begin{array}{l}\text { anti-bacterial, anti- } \\
\text { hypertensive, anti-diabetic, } \\
\text { anti-inflammatory, anti- } \\
\text { Parkinson, anti-ulcer, } \\
\text { spasmolytic, anti- } \\
\text { depressant }\end{array}$ & Havsteen B et al \\
\hline $\begin{array}{l}\text { Resveratrol } \\
(3,4,5- \\
\text { trihydroxystilb } \\
\text { ene) }\end{array}$ & $\begin{array}{l}\text { mainly found in } \\
\text { grapevines and } \\
\text { berries }\end{array}$ & $\begin{array}{l}\text { reducing oxidative } \\
\text { stress and advanced } \\
\text { glycation end-product } \\
\text { (AGE) production }\end{array}$ & $\begin{array}{l}\text { stimulating autophagy, } \\
\text { inhibiting endoplasmic } \\
\text { reticulum (ER) stress and } \\
\text { inflammation, ameliorating } \\
\text { lipotoxicity, activating the } \\
\text { AMP kinase (AMPK) } \\
\text { pathway, and modulating } \\
\text { angiogenesis }\end{array}$ & Evans JL et al \\
\hline
\end{tabular}




\begin{tabular}{|c|c|c|c|c|}
\hline Curcumin & $\begin{array}{l}\text { extracted from } \\
\text { the rhizome } \\
\text { Curcuma longa }\end{array}$ & $\begin{array}{l}\text { activates various } \\
\text { transcription factors, } \\
\text { such as NF-E2-related } \\
\text { factor (Nrf2),per - } \\
\text { oxisome proliferator- } \\
\text { activated receptor-g } \\
\text { (PPAR-g), CCAAT/ } \\
\text { enhancer binding } \\
\text { protein (C/EBP) } \\
\text { homologous protein } \\
\text { (CHOP) }\end{array}$ & $\begin{array}{l}\text { treat diabetic nephropathy, } \\
\text { microangiopathy and } \\
\text { retinopathy }\end{array}$ & $\begin{array}{l}\text { Khajehdehi et al } \\
\underline{221}\end{array}$ \\
\hline $\begin{array}{l}\text { Vitamin C } \\
\text { (ascorbic acid) }\end{array}$ & $\begin{array}{l}\text { Citrus fruits and } \\
\text { vegetables like } \\
\text { lemon, orange } \\
\text { etc. }\end{array}$ & $\begin{array}{l}\text { chain-breaking } \\
\text { antioxidant, } \\
\text { scavenging ROS } \\
\text { directly, and } \\
\text { preventing the } \\
\text { propagation of chain } \\
\text { reactions }\end{array}$ & $\begin{array}{l}\text { decreasing lipid } \\
\text { peroxidation and } \\
\text { augmented the activities of } \\
\text { antioxidant enzymes, SOD, } \\
\text { and GPx and reduced } \\
\text { albuminuria }\end{array}$ & Riccioni $\mathrm{G}$ et al \\
\hline
\end{tabular}

TGs $=$ Triglycerides $; \mathrm{ROS}=$ reactive oxygen species; $\mathrm{SCFA}=$ short chain fatty acid $; \mathrm{ER}=$ endoplasmic reticulum; $\mathrm{AMP}=$ adenosine monophosphate; $\mathrm{Nrf} 2=$ nuclear factor erythroid-2- related factor; $\mathrm{SOD}=$ superoxide dismutases; $\mathrm{GPx}=$ glutathione peroxidase

\section{Dietary fibers}

The term "dietary fiber" refers to a wide variety of natural food sources, refined grains, and commercial nutritional supplements. Dietary fiber is "an edible part of a plant or similar carbohydrate that fights digestion and digestion in the small intestine through complete or partial fermentation in the human large intestine. It is obtained from plant products of polysaccharides, oligosaccharides, lignin and other substances [86, 87, and 88]. Pharmacological use of dietary fibers: increasing body weight, lowering blood cholesterol levels and / or lowering blood sugar levels, coronary artery disease, dyslipidemia and hypertension [86]. Several studies have shown that the US population and diabetics do not respond to adequate daily fiber intake from their diet [88]. Mechanism of action dietary fibers in case of diabetes nephropathy. Soluble fiber: has been linked to decreased postprandial glucose levels and increased insulin sensitivity in diabetics and healthy people. This effect is usually associated with the viscous and / or gelling properties of soluble fiber [87, 88]. Insoluble fiber: Does not significantly affect post-meal blood sugar. While, consuming soluble fiber does not reduce the risk of type 2 diabetes [60]. In contrast, consumption of insoluble dietary fiber was most closely associated with a reduced risk of diabetes. To study the effect of fiber on the development of experimental diabetic nephropathy, previously experimental in which streptozotocin to induce diabetes in C57BL / 6 wild and knockout mice lacking the gene encoding the G-protein coupled receptor GPR43 or GPR109A. Diabetic mice were randomly assigned a diet high in fiber, normal, less in fiber, or high in SCFA in their drinking water. They found proton magnetic resonance imaging techniques to determine the metabolic profile and 16S ribosomal RNA sequencing to determine the entire gut micro biota [89]. Diabetic mice fed a high-fiber diet were significantly less likely to develop diabetic nephropathy. Mild proteinuria, glomerular hypertrophy, podocyte injury, and interstitial fibrosis were compared with control diabetic patients who received a normal or fiberfree diet. Dietary fiber promotes the proliferation of SCFA-producing bacteria from the genus Prevotella and Bifid bacterium, positively altering the microbial ecology of the intestine and improving dysbiosis by increasing the levels of SCFA in the faeces and the system. Reduction of fiber 
expression of genes encoding inflammatory cytokines, chemokine's, and proteins that induce fibrosis in diabetic kidney. Diabetic mice treated with SCFA were protected from nephropathy, but not in the absence of GPR43 or GPR109A. SCFA in vitro regulates inflammation of renal tubule cells and paw cells under hyperglycemic condition [89].

\section{Quercetin}

Quercetin is a flavonoid compound and it is belonging to a group of plant derived heterocyclic polyphenols [90]. It is obtained from in large amount of Cabbage, onions, fruits, apple, red wine, broccoli, cherries, tea and red wine etc. [91, 92]. Recent research shows that biochemical and pharmacological studies of Quercetin have shown that it is a potent ROS scavenger and may reduce the risk of cardiovascular disease and kidney disease [90, 93-95]. Quercetin also prevents various diseases such as osteoporosis, some forms of cancer, tumors, lungs, and diabetes mellitus. The antioxidant properties of quercetin play an important role in the prevention and treatment of these diseases [96]. Mechanism of action quercetin in case of Diabetes nephropathy (DN): Accumulating evidence suggests that increased oxidative stress may play an important role in the pathogenesis of DN [97, 98], but antioxidant therapy in diabetic patients has shown conflicting results during DN treatment [99]. Currently, only blockers of the renin-angiotensin system are chronically used. However, this mechanism has limitations [99,100], which justify the search for safer and more effective candidates for antioxidants. Flavonoids and their relative product i.e., quercetin have powerful antioxidant properties and are plant derived phenolic compounds found throughout the plant kingdom. Hence, Flavonoids are a therapeutic option [101,102]. Collected 8-week-old homozygous mice C57BL / 6J. Animals were placed at room temperature $\left(22^{\circ} \mathrm{C}\right)$ in a controlled humidity environment with a 12 hour / 12-hour dark light cycle in a laboratory translational physiology laboratory apparatus. Diabetic mice by intraperitoneal injection of STZ, diluted in citrate buffer (10 $\mathrm{mm}, \mathrm{pH} 4.5$ ) at a dose of $100 \mathrm{mg} / \mathrm{kg} /$ day for 3 days. Controls (non-diabetic, ND, $\mathrm{n}=10$ ) were given an equivalent volume of citrate buffered vehicle. Blood glucose was measured after food shortage for 6 hours after 1 week of STZ injection, and animals with glucose levels of $250 \mathrm{mg} / \mathrm{dL}$ or more for a minimum of 2 days were considered diabetic. Six weeks after STZ injection, diabetic mice were randomized to receive no treatment (soybean oil carrier, $\mathrm{DV}, \mathrm{n}=10$ ) or oral quercetin $(\mathrm{DQ}, \mathrm{n}=10$ ) at $10 \mathrm{mg} / \mathrm{kg}$ per day for 4 weeks. This dosage is based on previous studies on hypertension and diabetics animals [103,104]. This study suggests that chronic oral treatment with low doses of quercetin has an antidiabetic effect and attenuates the development of nephropathy in STZ-induced DN mice. These results are supported by reductions in plasma glucose, creatinine, triglycerides, proteinuria, and decreased interstitial matrix proliferation that accompanies. $\mathrm{O} 2$ production and apoptosis in kidney cells. In mice injected with STZ, $\beta$-cell damage and a decrease in the ability to secrete insulin were found [105,106]. These results support the role of oxidative stress in the development of diabetic nephropathy and suggest a possible antioxidant mechanism responsible for the nephroprotective effect of quercetin.

\section{Alpha lipoic acid [ALA]}

Alpha lipoic acid (ALA) is an anti-oxidant which occurs naturally in foods covalently bound to lysine in proteins [107]. Alpha lipoic acid is of two types: one is the R- alpha lipoic acid and the other is Salpha lipoic acid. The $\mathrm{R}$-isomer is present in nature and $\mathrm{S}$-isomer is not present in nature [108]. The most copious plant source of ALA is spinach, then broccoli, tomatoes, garden peas and rice bran [109]. Extreme concentrations of ALA are observed in animal tissues with immense metabolic action namely heart, liver and kidney. Contrary to ALA in foods, ALA in supplements is unbound to protein. 
Furthermore, the proportion of ALA available in dietary supplements $(200-600 \mathrm{mg}$ ) is expected to be 1000 times greater as compared to the proportion that could be acquired from the diet [107]. Four antioxidant characteristics of ALA have been analyzed: it's metal chelating activity; it's potential to scavenge reactive oxygen species (ROS); it's capacity to renew endogenous antioxidants and its property to restore oxidative destruction [110].ALA is clinically used in the treatment of Alzheimer's disease, cancer, diabetic complications, multiple sclerosis, diabetes, obesity and also it is used as an antioxidant [111].Oxidative stress seems to depict a chief role as a rapid development factor in diabetic nephropathy (DN) and later advancement [112]. ALA has powerful ROS-scavenging capacity. It has the exceptional attribute of being a ROS scavenger in its oxidized condition, quenching several radicals. ALA and dihydrolipoic acid(DHLA) together work as a redox couple (an electron donating molecule and its oxidized form), and have additional antioxidant characteristics comprising chelation of transition metals and the renewal of other antioxidants glutathione, vitamin $\mathrm{C}$, and vitamin E, for instance [7,8].ALA serve as a helper molecule for Various key mitochondrial multienzyme complexes, Improve the consumption of glucose by the cells, and modify the action of numerous signaling molecules and transcription elements. ALA and its derivative, DHLA, have a Straight antioxidant property owing to the neutralization of reactive oxygen species that are devastating to DNA, proteins and lipids of cells [115].Oxidative stress gives rise to endothelial cell (EC) damage and vascular dysfunction [116].Open and non-randomized trials in 84 diabetic sufferers. In this study, 49 patients (34 with type 1diabetes and 15 with type 2 diabetes) do not undergo antioxidant therapy and we're said to be standard. The remaining 35 patients ( 20 with type 1 diabetes and 15 with type 2 diabetes) Undergo ALA therapy ( $600 \mathrm{mg} /$ day for 18 months). The development of EC Destruction with regards to the evaluation of plasma thrombomodulin was remarkably increased in the standard group and reduced in the ALA therapy group after 18 months of research. However, the advancement of DN, as estimated by urinary albumin level, was considerably elevated in standard, but remains unaffected in the treated group. [117]

\section{Vitamin D}

Vitamin D (VD) is very uncommon in unfortified foods. VD is classified in two categories: vitamin $\mathrm{d} 2$ and vitamin $\mathrm{d} 3$. VD in different proportions is present in the flesh of fatty fish and oils of fish as well as cod and tuna liver oil $[118,119]$. Various foods are vitalized with Involving milk, few cereals and few bread items. There is preparatory authentication to indicate that meats from poultry, pork and beef comprise little proportion of VD that possibly eventuates from the VD that was vitalized in the animal feed [120]. Milk is also regarded as to be the food source of VD [121]. The vital source of VD for nearly all human beings is natural subjection to sunlight. It is determined that $80-90 \%$ of the body's demand for VD is fulfilled from this source [119]. Vitamin D receptor (VDR) emerges in various tissues and cells in the body [122,123]. 1,25(OH)2D has a broad extent of pharmacological Properties, namely hindrance of cellular proliferation and causing terminal differentiation, hindrance of angiogenesis, encouraging insulin production, restrict renin production, and stimulating macrophage cathelicidin development[122-125]. The deprivation of VD is the root cause of numerous chronic disorders like osteoporosis, fractures, diabetes, autoimmune disease, hypertension, periodontal disease, multiple sclerosis, cognitive impairments, Parkinson's disease, osteoarthritis, bacterial vaginitis. So, to prohibit all these disorders, the level of vitamin $d$ should be accordingly maintained [126].VD, a type of steroid having operative form $1,25(\mathrm{OH})_{2 \mathrm{D} 3}$, has been well known for the Significant functions in the modulation of serum calcium and phosphorus concentrations. It provides salient features by binding with its receptor VDR.VDR, a transcription agent present at chromosome 12 comprising 9 axons, is distinct nonsteroid nuclear hormone receptor super family, which gets involved in transcriptional management of genes in tissue- and cell-specific ways. 
Increasing documentation have indicated that VD/VDR signaling pathway procure a range of renalprotective outcomes in DN patients, including proteinuria, antifibrosis, anti-inflammatory, and Inhibiting podocyte devastation [127]. Animal studies have revealed that serum $25(\mathrm{OH}) \mathrm{D}$ (calcifediol) and 1, 25(OH) 2D3 (calcitriol) levels were considerably diminished in the DN rats and VD therapy of the rat model efficaciously prevented hyperglycemia-induced meningeal cell proliferation [128]. In one more animal study, diabetic VDR-/- mice exhibited more acute kidney injury and were expected to have severe proteinuria and glomerulosclerosis in contrast to the wildtype mice [129].

\section{Flavonoids}

In nature, Flavonoids compounds are products obtained from plants and they are detected in various parts of the plant. Flavonoids are utilized by vegetables for their development and Protection against plaque [130]. Plant based food and beverages comprise of plentiful of flavonoids namely fruits, vegetables, tea, cocoa and wine; therefore, they are named as dietary flavonoids. Flavonoids have various subdivisions, which contain chalcones, flavones, flavanols and isoflavones. These subdivisions have distinctive principal sources; onions and tea are chief dietary sources of flavanols and flavones, for instance [131]. Beside various biological properties in plants (resistance to herbivores, UV radiation and pathogens), flavonoids carry out countless pharmacological properties viz; anti-Parkinson, anti-ulcer, spasmolytic, anti-depressant, anti-bacterial, anti-hypertensive, antidiabetic, anti-inflammatory and anti-cancer [132]. New insights are proposed for the probable utilization of flavonoid derivatives as beneficial medicine to control specific cancers [133]. Flavonoids also provide auspicious benefits obesity- and inflammation-as well as COVID-19[133]. Scientific has strongly claimed that regular consumption of dietary flavonoids in potent amounts minimize the possibility of oxidative stress and chronic inflammation-mediated pathogenesis of human diseases such as CV disorder, specific cancers, neurological diseases and nephropathy [134]. Diabetic nephropathy (DN) is one of the most severe complications in DM, and it could be the reason of end-stage kidney disorder among sufferers which underwent chronic haemodialysis treatment [135]. In combination with a good hypoglycemic activity, flavonoids can be used to inhibit the occurrence and advancement of nephropathy [136]. The flavonoid mixture silymarin was estimated for its effectiveness on oxidative stress and morphology of kidney tissue in alloxan-induced diabetic rats. Tissue Devastation due to alloxan was inhibited by silymarin therapy and the activity and gene expression of three antioxidant enzymes were reinstated after the therapy with silymarin [136]. In further investigation, the preventive effect of silybin against high glucose-induced podocyte injury was discovered manifesting its Use against DN [137]. Countless investigations have illustrated antioxidant effects of various flavonoids on kidney. Depleted oxidative stress load and lessened renal devastation in a type 2 diabetic rat model by increasing the activity of the antioxidant enzymes Superoxide dismutase (SOD) and catalase (CAT), accompanied by introduction of the protective molecule Sirtuin-1[138]. Diosmetin, a flavonoid extracted from the leaves of oleaeuropaea with powerful antioxidant property, reinstated the lower levels of SOD and Nitric Oxide (NO) observed in the kidney of diabetic rats and diminished the levels of malondialdehyde (MDA), a lipid peroxidation marker [139].

\section{Curcumin}

Turmeric or Curcuma longa is a natural product, with a wide variety of therapeutic effects on several diseases such as neurodegenerative, hepatic and renal damage, cancer, and diabetes have been mainly attributed to its curcuminoid content. It is a bright yellow colored component extracted from the 
rhizome Curcuma longa, commonly known as turmeric [140]. The current basic evidences about the potential of curcumin/curcuminoids for the treatment of diabetes mellitus, mainly by its hypoglycemic, antioxidant, and anti-inflammatory properties [141]. Modern scientific studies shows that Curcumin is a highly pleiotropic molecule that interacts with numerous molecular targets, which it can up- or down regulate, Curcumin activates various transcription factors, such as NF-E2-related factor (Nrf2), peroxisome proliferator-activated receptor-g (PPAR-g), CCAAT/ enhancer binding protein (C/EBP) homologous protein (CHOP) and activating transcription factor 3 and it also been shown to down regulate various transcription factors, including NF-kB, protein kinases, chemokine's and inflammatory biomarkers [142] . A study showed that curcumin increases the half-life, mean residence time, and the apparent volume of distribution at steady state of glibenclamide in rats that may be due to decreased metabolism of the drug mediated by the inhibition of intestinal and hepatic CYP3A4 [143]. Despite the potential tremendous benefits, the clinical trials of curcumin are only available in using curcumin to treat diabetic nephropathy, microangiopathy and retinopathy so far. Clinical trials with curcumin in patients with overt type 2 DN (Diabetic nephropathy), a curcumin supplementation at a dose of $66.3 \mathrm{mg}$ per day for 2 months attenuated proteinuria, TGF-b and IL-8 [144]. Curcumin supplementation at the same dose has also been proved to decrease proteinuria, hematuria and systolic blood pressure in patients with relapsing or refractory lupus nephritis, which suggests that it can be used as an adjuvant safe therapy for such patients [145]. Further, there are several approaches to overcome limited solubility and poor bioavailability, these include encapsulation in various nanoparticles, such as methionine-dehydrophenylalanine nanoparticles, Ntrimethyl chitosan chloride-coated liposomes, chitosan micro particles and in Nano suspension emulsions, and sustained released tablets [146-148].

\section{Vitamin C (ascorbic acid)}

Vitamin $\mathrm{C}$ is also known as ascorbic acid, and act as a cofactor in multiple enzymatic reactions including collagen synthesis. $\underline{8}$ Citrus fruits and vegetables are the major source for Vitamin $\mathrm{C}$ such as orange sand orange juice, strawberries, blackcurrants,peppers, , broccoli, Brussels sprouts, potatoes. For adults the recommended dietary intake of vitamin $\mathrm{C}$ is $45 \mathrm{mg}$ per day. $1 \mathrm{~A}$ wide variety of disorders like diabetes, atherosclerosis, common cold, cataracts, glaucoma, macular degeneration, stroke, heart diseases, and cancer and so on, can be treated and prevented by Vitamin C. Vit. C is a chain-breaking antioxidant, scavenging ROS directly, and preventing the propagation of chain reactions that would otherwise show reduction in protein Glycation [149]. The diabetic condition possibly causes a variety of tissue damage in patients with diabetes and also production of oxidative stress. And further, the increased oxidative stress in the diabetic kidney may induce apoptosis, which may contribute to the development of diabetic nephropathy [150,151]. Vitamin C plays a crucial role in the antioxidant defense system and in the apoptosis. It was demonstrated that vitamin $\mathrm{C}$ exclusion from tubular epithelial cells, through the competition of glucose and dehydroascorbate for a common transport mechanism, will deprive the cells of antioxidant ability and could lead to ROS accumulation in diabetes [152]. It was demonstrated that in diabetic rats the administration of vitamin $\mathrm{C}$ can protect podocyte by increasing antioxidative capacity and ameliorating the renal OS [153,154]. In addition, it also acts by decreasing lipid peroxidation and augmented the activities of antioxidant enzymes, SOD, CAT, and GPx and reduced albuminuria, and GBM thickness in the kidneys of diabetic rats [155]. It was found that vitamin $\mathrm{C}$ protects renal lesions in DN by inhibiting expression of type IV collagen [156]. It has been shown that via Nrf2 activation vitamin C increases HO-1 protein expression in a concentration- and time-dependent manner. In animals, it brings down the diabetes-induced sorbitol accumulation and lipid peroxides in erythrocytes [149]. When vitamin C is combined with metformin a significant reduction in $\mathrm{HbAlc}$ levels in diabetic patients observed as compared to metformin alone 
[157]. Another study showed that administration of vitamin $C$ with antidiabetic drugs enhanced antioxidant defenses and reduced oxidative damage [158].

\section{Resveratrol}

Resveratrol (3, 4', 5-trihydroxystilbene) is a nutraceutical that is mainly found in grapevines and berries and available in various pharmaceutical dosages. It is a phytoalexin, firstly isolated from Veratrum grandiflorum. Resveratrol has nephroprotective effects through various mechanisms including reducing oxidative stress and advanced Glycation end-product (AGE) production, stimulating autophagy, inhibiting endoplasmic reticulum (ER) stress and inflammation, ameliorating lipotoxicity, activating the AMP kinase (AMPK) pathway, and modulating angiogenesis that eventually help in maintaining optimum kidney function during DN $[159,160]$. Moreover, the use of resveratrol as an adjuvant to conventional antidiabetic therapies could be an effective approach to manage DN. Previous reports suggest that elevated glucose flux can increase reactive oxygen species (ROS) production by the mitochondrial electron transport chain [161]. The accumulation of ROS can overwhelm the cellular antioxidant defense system and thus induce renal damage via different mechanisms, including activation of the polyol pathway and Hexosamine biosynthesis pathway, AGEs production, and activation of protein kinase C (PKC) [159,160]. Therefore, natural products like resveratrol with potential antioxidant capacity can attenuate these pathways and the associated complications for the control of DN. It has been founded that ER stress is one of the major contributing factors in the onset and progression of various pathological conditions including DN [162]. In addition, Resveratrol also acts by inhibition of renal epidermal growth factor receptors (EGFRs) and reduce ER stress in DN [163]. Pre-clinical studies shows that Resveratrol treatment [5 and $10 \mathrm{mg} /(\mathrm{kg} \cdot \mathrm{d})]$ in a diabetic rat model (streptozotocin-induced DM), significantly attenuated pre-DN symptoms including reduced clearance of creatinine and urea, proteinuria, and oxidative stress (elevated lipid peroxidation and decreased antioxidant enzyme activity) via its antioxidant activity and resveratrol treatment $[5 \mathrm{mg} /(\mathrm{kg} \cdot \mathrm{d})]$ in type 1 diabetes rats significantly improve renal hypertrophy and structural changes, including tubular atrophy, meningeal expansion, diffused glomerulonephritis, and renal fibrosis. Preliminary human studies confirm the effectiveness of Resveratrol as given: - 613 type 2 diabetes confirm the effectiveness of this compound, 614 particular improves glycaemic control and 615 decreases insulin resistance $[134,135,136]$.

Conclusion: Among many disease or disorders of carbohydrate, fat and protein metabolism, diabetes is most serious disorder effecting large population of the world. It is allied with decreased insulin production or resistance towards its action. The routine medical treatments used for DM are notenough therapeutic effective and have many undesirable side effects. Moreover, the global increased dispersal of DM makes researchers try to find out potential complementary or alternative treatments.Plants can be used to treat diabetes patients, both insulin dependent \& non-insulin dependent diabetes. Nutraceuticals are the food supplements having nutritional value. All the nutrients discussed in this review have shown significant clinical \& pharmacological activity. The potency of herbal drugs is prominent \& they have very less side effects than the synthetic anti-diabetic drugs. There is increasing demand by patients to use the natural products with anti-diabetic activity. This review summarize the role of nutraceuticals in diabetic nephropathy (DN). The nutraceuticals including omega-3- fatty acid, vitamin D, dietary fibers, curcumin, resveratrol, quercetin, berberine, vitamin $\mathrm{C}$ have beneficial effects on pathophysiology of DN. Most of the nutraceuticals used in clinical practicemodulate one or more pathogenic mechanismsunderlying the progress of diabetes mellitus, metabolic syndrome, and their complications. 


\section{References}

1. Guyton, Arthur C., and John E. Hall. "Textbook of medical physiology, 11th." WB Sounders Company, Philadelphia (2006).

2. Atlas, Diabetes. "International diabetes federation." IDF Diabetes Atlas, 7th edn. Brussels, Belgium: International Diabetes Federation (2015).

3. Fong, Donald S., et al. "Retinopathy in diabetes." Diabetes care 27.suppl 1 (2004): s84-s87.

4. Brorsson, Anna Lena, et al. "FDA USA OKs First-Ever Nasal Glucagon for Severe Hypos in Diabetes." mars 2019 (2019).

5. Bhattacharjee, Niloy, et al. "Mechanistic insight of diabetic nephropathy and its pharmacotherapeutic targets: an update." European journal of pharmacology 791 (2016): 8-24.

6. Evans, Katharine, et al. "UK renal registry 20th annual report: introduction." Nephron 139 (2018): 1-12.

7. Parving, Hans-Henrik, et al. "Prevalence of microalbuminuria, arterial hypertension, retinopathy, and neuropathy in patients with insulin dependent diabetes." Br Med J (Clin Res Ed) 296.6616 (1988): 156-160.

8. Rossing, Peter, Philip Hougaard, and Hans-Henrik Parving. "Progression of microalbuminuria in type 1 diabetes: ten-year prospective observational study." Kidney international 68.4 (2005): 1446-1450.

9. Umanath, Kausik, and Julia B. Lewis. "Update on diabetic nephropathy: core curriculum 2018." American Journal of Kidney Diseases 71.6 (2018): 884-895

10. Zhao, Jian. "Nutraceuticals, nutritional therapy, phytonutrients, and phytotherapy for improvement of human health: a perspective on plant biotechnology application." Recent patents on biotechnology 1.1 (2007): 75-97.

11. Sheira, Gehan, et al. "Urinary biomarker N-acetyl- $\beta$-D-glucosaminidase can predict severity of renal damage in diabetic nephropathy." Journal of Diabetes \& Metabolic Disorders 14.1 (2015): 1-5.

12. Ramana, Kota V. "Aldose reductase: new insights for an old enzyme." (2011): 103-114.

13. Tadege, Million, and Kirankumar S. Mysore. "Tnt1 retrotransposon tagging of STF in Medicago truncatula reveals tight coordination of metabolic, hormonal and developmental signals during leaf morphogenesis." Mobile genetic elements 1.4 (2011): 301-329.

14. Kage-Nakadai, Eriko, Tomoko Uehara, and Shohei Mitani. "H+/myo-inositol transporter genes, hmit-1.1 and hmit1.2, have roles in the osmoprotective response in Caenorhabditis elegans." Biochemical and biophysical research communications 410.3 (2011): 471-477.

15. Bonventre, Joseph V. "Can we target tubular damage to prevent renal function decline in diabetes?." Seminars in nephrology. Vol. 32. No. 5. WB Saunders, 2012.

16. Ishibashi, Yuji, et al. "Pravastatin inhibits advanced glycation end products (AGEs)-induced proximal tubular cell apoptosis and injury by reducing receptor for AGEs (RAGE) level." Metabolism 61.8 (2012): 1067-1072.

17. Lee, Sang-Hun, et al. "The protective effect of Salvia miltiorrhiza in an animal model of early experimentally induced diabetic nephropathy." Journal of ethnopharmacology 137.3 (2011): 1409-1414

18. Ha, Yonju, et al. "Diabetes accelerates retinal ganglion cell dysfunction in mice lacking sigma receptor 1." Molecular vision 18 (2012): 2860

19. Drobyshevsky, Alexander, et al. "Antenatal insults modify newborn olfactory function by nitric oxide produced from neuronal nitric oxide synthase." Experimental neurology 237.2 (2012): 427-434.

20. Liu, I-Min, et al. "Angelica Acutiloba Root Alleviates Advanced Glycation End-Product-Mediated Renal Injury in Streptozotocin-Diabetic Rats." Journal of food science 76.7 (2011): H165-H174.

21. Lattke, Michael, et al. "Nuclear factor $\mathrm{kB}$ activation impairs ependymal ciliogenesis and links neuroinflammation to hydrocephalus formation." Journal of Neuroscience 32.34 (2012): 11511-11523.

22. Song, Jin, et al. "Involvement of protein kinase C-CPI-17 in androgen modulation of angiotensin II-renal vasoconstriction." Cardiovascular research 85.3 (2010): 614-621.

23. Schwartz, Idit F., et al. "Attenuated glomerular arginine transport prevents hyperfiltration and induces HIF-1 $\alpha$ in the pregnant uremic rat." American Journal of Physiology-Renal Physiology 303.3 (2012): F396-F404.

24. Cui, Bei, et al. "Aquaporin 4 knockdown exacerbates streptozotocin-induced diabetic retinopathy through aggravating inflammatory response." Experimental eye research 98 (2012): 37-43.

25. Singh, Vivek P., Kenneth M. Baker, and Rajesh Kumar. "Activation of the intracellular renin-angiotensin system in cardiac fibroblasts by high glucose: role in extracellular matrix production." American Journal of PhysiologyHeart and Circulatory Physiology 294.4 (2008): H1675-H1684. 
26. Zheng, J-M., et al. "Rhein reverses the diabetic phenotype of mesangial cells over-expressing the glucose transporter (GLUT1) by inhibiting the hexosamine pathway." British journal of pharmacology 153.7 (2008): 14561464.

27. Navarro-González, Juan F., et al. "Inflammatory molecules and pathways in the pathogenesis of diabetic nephropathy." Nature Reviews Nephrology 7.6 (2011): 327-340.

28. Murray, Katie N., Adrian R. Parry-Jones, and Stuart M. Allan. "Interleukin-1 and acute brain injury." Frontiers in cellular neuroscience 9 (2015): 18.

29. Gordon, C., et al. "Urinary IL-6: a marker for mesangial proliferative glomerulonephritis?." Clinical \& Experimental Immunology 86.1 (1991): 145-149.

30. Gheith, Osama, et al. "Diabetic kidney disease: difference in the prevalence and risk factors worldwide." Journal of The Egyptian Society of Nephrology and Transplantation 16.3 (2016): 65.

31. Nemeth, Elizabeta, et al. "Hepcidin regulates cellular iron efflux by binding to ferroportin and inducing its internalization." science 306.5704 (2004): 2090-2093.

32. Fiseha, Temesgen. "Urinary biomarkers for early diabetic nephropathy in type 2 diabetic patients." Biomarker research 3.1 (2015): 1-7.

33. Wright, Ernest M., Donald DF Loo, and Bruce A. Hirayama. "Biology of human sodium glucose transporters." Physiological reviews 91.2 (2011): 733-794.

34. Ahn, Jae Hee, et al. "Effect of eplerenone, a selective aldosterone blocker, on the development of diabetic nephropathy in type 2 diabetic rats." Diabetes \& metabolism journal 36.2 (2012): 128-135.

35. Lai, Lingyun, et al. "Aldosterone promotes fibronectin production through a Smad2-dependent TGF- $\beta 1$ pathway in mesangial cells." Biochemical and biophysical research communications 348.1 (2006): 70-75.

36. Buffon, Marjoriê P., et al. "rs1888747 polymorphism in the FRMD3 gene, gene and protein expression: role in diabetic kidney disease." Diabetology \& metabolic syndrome 8.1 (2016): 1-10.

37. Martini, Sebastian, et al. "From single nucleotide polymorphism to transcriptional mechanism: a model for FRMD3 in diabetic nephropathy." Diabetes 62.7 (2013): 2605-2612.

38. Palmer, Nicholette D., and Barry I. Freedman. "FRMD3 in diabetic nephropathy—guilt by association." Nature Reviews Nephrology 9.6 (2013): 313-314.

39. Zeisberg, Michael, and Raghu Kalluri. "Reversal of experimental renal fibrosis by BMP7 provides insights into novel therapeutic strategies for chronic kidney disease." Pediatric nephrology 23.9 (2008): 1395-1398.

40. Liu R, Lee K, He JC. "Genetics and epigenetics of diabetic nephropathy.” Kidney Disease 2015;1:42e51.

41. Wang, Shinong, et al. "Renal bone morphogenetic protein-7 protects against diabetic nephropathy." Journal of the American Society of Nephrology 17.9 (2006): 2504-2512.

42. Clark, William F., et al. "Fish oil in lupus nephritis: clinical findings and methodological implications." Kidney international 44.1 (1993): 75-86.

43. Chewcharat, Api, et al. "The effects of omega-3 fatty acids on diabetic nephropathy: A meta-analysis of randomized controlled trials." PloS one 15.2 (2020): e0228315.

44. Donadio Jr, James V., et al. "A controlled trial of fish oil in IgA nephropathy." New England Journal of Medicine 331.18 (1994): 1194-1199.

45. Baggio, Bruno, Estella Musacchio, and Giovanna Priante. "Polyunsaturated fatty acids and renal fibrosis: pathophysiologic link and potential clinical implications." Journal of nephrology 18.4 (2005): 362.

46. Garman, Joseph H., et al. "Omega-3 fatty acid rich diet prevents diabetic renal disease." American Journal of Physiology-Renal Physiology 296.2 (2009): F306-F316.

47. Domitrović, Robert, et al. "Berberine exerts nephroprotective effect against cisplatin-induced kidney damage through inhibition of oxidative/nitrosative stress, inflammation, autophagy and apoptosis." Food and Chemical Toxicology 62 (2013): 397-406.

48. Han, Junling, Huiling Lin, and Weiping Huang. "Modulating gut microbiota as an anti-diabetic mechanism of berberine." Medical science monitor: international medical journal of experimental and clinical research 17.7 (2011): RA164.

49. Imenshahidi, Mohsen, and Hossein Hosseinzadeh. "Berberis vulgaris and berberine: an update review." Phytotherapy research 30.11 (2016): 1745-1764.

50. Mohammadzadeh, Nooshin, Soghra Mehri, and Hossein Hosseinzadeh. "Berberis vulgaris and its constituent berberine as antidotes and protective agents against natural or chemical toxicities." Iranian journal of basic medical sciences 20.5 (2017): 538.

51. Wang, Y. Y., Q. Liu, and L. Q. Tang. "Effect of berberine on high glucose induced podocyte functions and related protein expression." Chin Pharmacol Bull 34.8 (2018): 1158-1163.

52. Qiu, Yuan-Ye, Li-Qin Tang, and Wei Wei. "Berberine exerts renoprotective effects by regulating the AGEsRAGE signaling pathway in mesangial cells during diabetic nephropathy." Molecular and cellular endocrinology 443 (2017): 89-105.

53. Tang, Li-Qin, et al. "Renoprotective effects of berberine and its potential effect on the expression of $\beta$-arrestins and intercellular adhesion molecule-1 and vascular cell adhesion molecule-1 in streptozocin-diabetic nephropathy rats." Journal of diabetes 8.5 (2016): 693-700.

54. Sun, Si-Fan, et al. "Renoprotective effect of berberine on type 2 diabetic nephropathy in rats." Clinical and Experimental Pharmacology and Physiology 42.6 (2015): 662-670.

55. JULIE M, Jones. "The definition of dietary fibers." Cereal foods world 46.3 (2001): 112-129. 
56. Anetor, J. I., et al. "Decreased serum magnesium and zinc levels: atherogenic implications in type-2 diabetes mellitus in Nigerians." Nutrition and health 16.4 (2002): 291-300.

57. Papathanasopoulos, Athanasios, and Michael Camilleri. "Dietary fiber supplements: effects in obesity and metabolic syndrome and relationship to gastrointestinal functions." Gastroenterology 138.1 (2010): 65-72.

58. Li, Yan Jun, et al. "Dietary fiber protects against diabetic nephropathy through short-chain fatty acid-mediated activation of G protein-coupled receptors GPR43 and GPR109A." Journal of the American Society of Nephrology 31.6 (2020): 1267-1281.

59. Rauf, Abdur, et al. "Anticancer potential of quercetin: A comprehensive review." Phytotherapy Research 32.11 (2018): 2109-2130.

60. Almeida, A. Filipa, et al. "Bioavailability of quercetin in humans with a focus on interindividual variation." Comprehensive reviews in food science and food safety 17.3 (2018): 714-731.

61. David, Alexander Victor Anand, Radhakrishnan Arulmoli, and Subramani Parasuraman. "Overviews of biological importance of quercetin: A bioactive flavonoid." Pharmacognosy reviews 10.20 (2016): 84.

62. Perez-Vizcaino, Francisco, et al. "Antihypertensive effects of the flavonoid quercetin." Pharmacological Reports 61.1 (2009): 67-75.

63. Bischoff, Stephan C. "Quercetin: potentials in the prevention and therapy of disease." Current Opinion in Clinical Nutrition \& Metabolic Care 11.6 (2008): 733-740.

64. Coqueiro, Aline, et al. "Free radical scavenging activity of Kielmeyera variabilis (Clusiaceae)." Molecules 18.2 (2013): 2376-2385.

65. Boots, Agnes W., Guido RMM Haenen, and Aalt Bast. "Health effects of quercetin: from antioxidant to nutraceutical." European journal of pharmacology 585.2-3 (2008): 325-337.

66. Brosius, Frank C., et al. "Mouse models of diabetic nephropathy." Journal of the American Society of Nephrology 20.12 (2009): 2503-2512.

67. Brosius III, Frank C., and Charles E. Alpers. "New targets for treatment of diabetic nephropathy: what we have learned from animal models." Current opinion in nephrology and hypertension 22.1 (2013): 17.

68. Tavafi, Majid. "Diabetic nephropathy and antioxidants." Journal of nephropathology 2.1 (2013): 20.

69. Cooper, Mark E. "Interaction of metabolic and haemodynamic factors in mediating experimental diabetic nephropathy." Diabetologia 44.11 (2001): 1957-1972.

70. Cai, X., et al. "Bioavailability of quercetin: problems and promises." Current medicinal chemistry 20.20 (2013): 2572-2582.

71. Pérez-Gregorio, M. R., et al. "Increasing the added-value of onions as a source of antioxidant flavonoids: a critical review." Critical Reviews in Food Science and Nutrition 54.8 (2014): 1050-1062.

72. Machha, Ajay, and MohdRais Mustafa. "Chronic treatment with flavonoids prevents endothelial dysfunction in spontaneously hypertensive rat aorta." Journal of cardiovascular pharmacology 46.1 (2005): 36-40.

73. Ajay, Machha, et al. "Effect of quercetin on altered vascular reactivity in aortas isolated from streptozotocininduced diabetic rats." Diabetes Research and Clinical Practice 73.1 (2006): 1-7.

74. Like, Arthur A., and Aldo A. Rossini. "Streptozotocin-induced pancreatic insulitis: new model of diabetes mellitus." Science 193.4251 (1976): 415-417.

75. Wang, Chuang, et al. "Quercetin and allopurinol ameliorate kidney injury in STZ-treated rats with regulation of renal NLRP3 inflammasome activation and lipid accumulation." PloS one 7.6 (2012): e38285.

76. Hendler, S. S., and D. R. Rorvik. "PDR for nutritional supplements. Montvale: Medical Exonomics." (2001).

77. Laher, Issy. "Diabetes and alpha lipoic acid." Frontiers in pharmacology 2 (2011): 69.

78. Lodge, J. K., et al. "Natural sources of lipoic acid: determination of lipoyllysine released from protease-digested tissues by high performance liquid chromatography incorporating electrochemical detection." Journal of Applied Nutrition 49.1-2 (1997): 3-11.

79. Biewenga, Gerreke Ph, Guido RMM Haenen, and Aalt Bast. "The pharmacology of the antioxidant lipoic acid." General Pharmacology: The Vascular System 29.3 (1997): 315-331.

80. Smith, A. R., et al. "Lipoic acid as a potential therapy for chronic diseases associated with oxidative stress." Current medicinal chemistry 11.9 (2004): 1135-1146.

81. Fujita, Atsuyo, et al. "Ferulic acid prevents pathological and functional abnormalities of the kidney in Otsuka Long-Evans Tokushima Fatty diabetic rats." Diabetes research and clinical practice 79.1 (2008): 11-17.

82. Elliott, David B. "Systematic reviews of optometric interventions." Ophthalmic and Physiological Optics 32.3 (2012): 173

83. Singh, Uma, and IshwarlalJialal. "Retracted: alpha-lipoic acid supplementation and diabetes." Nutrition reviews 66.11 (2008): 646-657.

84. Tutelyan, Viktor A., et al. "Lipoic acid: physiological role and prospects for clinical application." Voprosypitaniia 88.4 (2019): 6-11.

85. Förstermann, Ulrich. "Oxidative stress in vascular disease: causes, defense mechanisms and potential therapies." Nature clinical practice Cardiovascular medicine 5.6 (2008): 338-349.

86. Morcos, M., et al. "Effect of $\alpha$-lipoic acid on the progression of endothelial cell damage and albuminuria in patients with diabetes mellitus: an exploratory study." Diabetes research and clinical practice 52.3 (2001): 175183.

87. Holick, Michael F., P. K. T. Pang, and M. P. Schreibman. "Phylogenetic and evolutionary aspects of vitamin D from phytoplankton to humans." Vertebrate endocrinology: fundamentals and biomedical implications 3 (1989): 7-43.

88. Holick, Michael F. "McCollum Award Lecture, 1994: vitamin D—new horizons for the 21st century." The American journal of clinical nutrition 60.4 (1994): 619-630. 
89. Thompson, J. N., and Louise Plouffe. "Determination of cholecalciferol in meat and fat from livestock fed normal and excessive quantities of vitamin D." Food chemistry 46.3 (1993): 313-318.

90. Chen, Tai C., et al. "An update on the vitamin D content of fortified milk from the United States and Canada." New England Journal of Medicine 329.20 (1993): 1507-1507.

91. Nair, Rathish, and Arun Maseeh. "Vitamin D: The "sunshine" vitamin." Journal of pharmacology \& pharmacotherapeutics 3.2 (2012): 118.

92. Chlebowski, Rowan T., et al. "Calcium plus vitamin D supplementation and the risk of breast cancer." JNCI: Journal of the National Cancer Institute 100.22 (2008): 1581-1591.

93. Stolzenberg-Solomon, Rachael Z., et al. "A prospective nested case-control study of vitamin D status and pancreatic cancer risk in male smokers." Cancer research 66.20 (2006): 10213-10219.

94. Stolzenberg-Solomon, Rachael Z., et al. "Serum vitamin D and risk of pancreatic cancer in the prostate, lung, colorectal, and ovarian screening trial." Cancer research 69.4 (2009): 1439-1447.

95. Holick, Michael F. "High prevalence of vitamin D inadequacy and implications for health." Mayo Clinic Proceedings. Vol. 81. No. 3. Elsevier, 2006.

96. Lei, Min, Zhangsuo Liu, and Jia Guo. "The Emerging Role of Vitamin D and Vitamin D Receptor in Diabetic Nephropathy." BioMed Research International 2020 (2020).

97. Wang, Hang, et al. "In vitro and in vivo inhibition of mTOR by 1,25-dihydroxyvitamin D 3 to improve early diabetic nephropathy via the DDIT4/TSC2/mTOR pathway." Endocrine 54.2 (2016): 348-359.

98. Zhang, Z., et al. "Renoprotective role of the vitamin D receptor in diabetic nephropathy." Kidney international 73.2 (2008): 163-171.

99. Havsteen, Bent H. "The biochemistry and medical significance of the flavonoids." Pharmacology \& therapeutics 96.2-3 (2002): 67-202.

100. Griesbach, R. J. "Biochemistry and genetics of flower color." Plant Breed Rev 25 (2005): 89-114.

101. Rana, Avtar Chand, and Bhawna Gulliya. "Chemistry and pharmacology of flavonoids-a review." Indian Journal of Pharmaceutical Education and Research 53.1 (2019): 8-20.

102. Rupasinghe, H. P. "Special issue "flavonoids and their disease prevention and treatment potential": recent advances and future perspectives." Molecules 25.20 (2020): 4746.

103. Williamson, Gary, Colin D. Kay, and Alan Crozier. "The bioavailability, transport, and bioactivity of dietary flavonoids: A review from a historical perspective." Comprehensive Reviews in Food Science and Food Safety 17.5 (2018): 1054-1112.

104. Patient Registration Committee, Japanese Society for Dialysis Therapy. "An overview of regular dialysis treatment in Japan as of 31 December 2003." Therapeutic Apheresis and Dialysis 9.6 (2005): 431-458.

105. Soto, C., et al. "Effect of silymarin on kidneys of rats suffering from alloxan-induced diabetes mellitus." Phytomedicine 17.14 (2010): 1090-1094.

106. Khazim, Khaled, et al. "The antioxidant silybin prevents high glucose-induced oxidative stress and podocyte injury in vitro and in vivo." American Journal of Physiology-Renal Physiology 305.5 (2013): F691-F700.

107. Oza, Manisha J., and Yogesh A. Kulkarni. "Formononetin attenuates kidney damage in type 2 diabetic rats." Life sciences 219 (2019): 109-121.

108. Jiang, Yingsong, et al. "Diosmetin attenuates Akt signaling pathway by modulating nuclear factor kappa-lightchain-enhancer of activated B cells (NF-kB)/inducible nitric oxide synthase (iNOS) in streptozotocin (STZ)induced diabetic nephropathy mice." Medical science monitor: international medical journal of experimental and clinical research 24 (2018): 7007.

109. Surh, Young-Joon, and Kyung-Soo Chun. "Cancer chemopreventive effects of curcumin." The molecular targets and therapeutic uses of curcumin in health and disease (2007): 149-172.

110. Lan, Tian, et al. "Sphingosine kinase-1 pathway mediates high glucose-induced fibronectin expression in glomerular mesangial cells." Molecular Endocrinology 25.12 (2011): 2094-2105.

111. Zhou, Hongyu, Christopher S Beevers, and Shile Huang. "The targets of curcumin." Current drug targets 12.3 (2011): 332-347.

112. Devi, PR Sakunthala, et al. "Pharmacokinetic interaction of curcumin and glibenclamide in diabetic rats." Veterinary world 8.4 (2015): 508.

113. Khajehdehi, Parviz, et al. "Oral supplementation of turmeric attenuates proteinuria, transforming growth factor- $\beta$ and interleukin-8 levels in patients with overt type 2 diabetic nephropathy: a randomized, double-blind and placebo-controlled study." Scandinavian journal of urology and nephrology 45.5 (2011): 365-370.

114. Khajehdehi, Parviz, et al. "Oral supplementation of turmeric decreases proteinuria, hematuria, and systolic blood pressure in patients suffering from relapsing or refractory lupus nephritis: a randomized and placebo-controlled study." Journal of Renal Nutrition 22.1 (2012): 50-57.

115. Alam, Shadab, Jiban J. Panda, and Virander S. Chauhan. "Novel dipeptide nanoparticles for effective curcumin delivery." International journal of nanomedicine 7 (2012): 4207.

116. Gao, Yan, et al. "In vivo evaluation of curcumin loaded nanosuspensions by oral administration." Journal of biomedical nanotechnology 8.4 (2012): 659-668.

117. Chen, Huanlei, et al. "N-trimethyl chitosan chloride-coated liposomes for the oral delivery of curcumin." Journal of liposome research 22.2 (2012): 100-109.

118. Riccioni, Graziano, et al. "Antioxidant vitamin supplementation in cardiovascular diseases." Annals of Clinical \& Laboratory Science 37.1 (2007): 89-95.

119. Zhang, Weiping, et al. "Diabetes-induced apoptosis in rat kidney." Biochemical and molecular medicine 61.1 (1997): 58-62. 
120. Ohno, Takamasa, et al. "Water extract of the root of Lindera strychnifolia slows down the progression of diabetic nephropathy in db/db mice." Life sciences 77.12 (2005): 1391-1403.

121. Chen, Ling, et al. "Hyperglycemia inhibits the uptake of dehydroascorbate in tubular epithelial cell." American journal of nephrology 25.5 (2005): 459-465.

122. Lee, Eun Young, et al. "Blockade of oxidative stress by vitamin $\mathrm{C}$ ameliorates albuminuria and renal sclerosis in experimental diabetic rats." Yonsei medical journal 48.5 (2007): 847-855.

123. Qin, Qiao-Jing, et al. "Study on the effect and mechanism of ascorbic acid on renal podocytes in diabetes." Zhongguoyingyong sheng li xue za zhi= Zhongguoyingyongshenglixuezazhi= Chinese journal of applied physiology 24.1 (2008): 112-115.

124. Kędziora-Kornatowska, K., et al. "Effect of vitamin E and vitamin C supplementation on antioxidative state and renal glomerular basement membrane thickness in diabetic kidney." Nephron Experimental Nephrology 95.4 (2003): e134-e143.

125. Li, Qiangxiang, et al. "Effects of aminoguanidine and vitamin C on collagen type IV in diabetic nephropathy rats." Endocrine 39.3 (2011): 251-258

126. Dakhale, Ganesh N., Harshal V. Chaudhari, and Meena Shrivastava. "Supplementation of vitamin C reduces blood glucose and improves glycosylated hemoglobin in type 2 diabetes mellitus: a randomized, double-blind study." Advances in pharmacological sciences 2011 (2011).

127. Das, Sushanta Kr, et al. "Antioxidant effect of vitamin $\mathrm{c}$ on type 2 diabetes mellitus patients along with two different oral hypoglycemic agents for smooth glycemic control." WJPPS 1 (2012): 1113-1122.

128. Evans, Joseph L., et al. "Oxidative stress and stress-activated signaling pathways: a unifying hypothesis of type 2 diabetes." Endocrine reviews 23.5 (2002): 599-622.

129. Giacco, Ferdinando, and Michael Brownlee. "Oxidative stress and diabetic complications." Circulation research 107.9 (2010): 1058-1070

130. Brownlee, Michael. "The pathobiology of diabetic complications: a unifying mechanism." diabetes 54.6 (2005): 1615-1625.

131. Lupachyk, Sergey, et al. "Endoplasmic reticulum stress plays a key role in the pathogenesis of diabetic peripheral neuropathy." Diabetes 62.3 (2013): 944-952.

132. Bhatt, Jayesh Kumar, Sabin Thomas, and Moola JogheeNanjan. "Resveratrol supplementation improves glycemic control in type 2 diabetes mellitus." Nutrition research 32.7 (2012): 537-541.

133. Louis, Xavier Lieben, et al. "Obesity and Cardiovascular Disease: Impact of Resveratrol as a Therapeutic." Biochemistry of Cardiovascular Dysfunction in Obesity. Springer, Cham, 2020. 283-305.

134. Movahed, Ali, et al. "Antihyperglycemic effects of short term resveratrol supplementation in type 2 diabetic patients." Evidence-Based complementary and alternative medicine 2013 (2013).

135. N.L. Price, A.P. Gomes, A.J. Ling, F.V. Duarte, A. Martin-Montalvo, B.J. North, B. 969 Agarwal, L. Ye, G. Ramadori, J.S. Teodoro, B.P. Hubbard, A.T. Varela, J.G. Davis, 970 B. Varamini, A. Hafner, R. Moaddel, A.P. Rolo, R. Coppari, C.M. Palmeira, R. de 971 Cabo, J.A. Baur, D.A. Sinclair, SIRT1 is required for AMPK activation and the 972 beneficial effects of resveratrol on mitochondrial function, Cell Metab. 15973 (2012) 675690. 974. 